\title{
Weight Changes Post-Phentermine Use
}

Cassandra S. Towns

Central Michigan University, steadmancassandra@yahoo.com

Roschelle A. Heuberger

Central Michigan University, heube1ra@cmich.edu

Jack Logomarsino

Central Michigan University, Jack.Logomarsino@cmich.edu

Arturo Olivera Jr.

Loyola University, aojr@aol.com

Follow this and additional works at: https://nsuworks.nova.edu/ijahsp

Part of the Medicine and Health Sciences Commons

This Manuscript has supplementary content. View the full record on NSUWorks here: https://nsuworks.nova.edu/ijahsp/vol18/iss2/4

\section{Recommended Citation}

Towns CS, Heuberger RA, Logomarsino J, Olivera Jr. A. Weight Changes Post-Phentermine Use. The Internet Journal of Allied Health Sciences and Practice. 2020 Jan 01;18(2), Article 4.

This Manuscript is brought to you for free and open access by the College of Health Care Sciences at NSUWorks. It has been accepted for inclusion in Internet Journal of Allied Health Sciences and Practice by an authorized editor of NSUWorks. For more information, please contact nsuworks@nova.edu. 


\title{
Weight Changes Post-Phentermine Use
}

\begin{abstract}
Purpose: Evaluate weight maintenance in subjects who lost weight while taking phentermine (PTM). Methods: This study was conducted by utilizing a validated survey to collect self-reported data about weight changes post-PTM use. The survey was sent to 19,986 email addresses of current and former patients of a PTM-based outpatient clinic, located in Chicago, IL; 546 participants completed the survey. Results were analyzed using a variety of techniques. Results: At the time of the survey, $42 \%$ of responders maintained all the weight lost while on PTM, and $25.2 \%$ had maintained a weight loss of at least $10 \%$ of their body weight. The average weight lost and maintained at the time of the survey was $3.75 \% \pm$ $13.8 \%$ of body weight or $8.74 \mathrm{lbs} \pm 27.45 \mathrm{lbs}$. Nineteen responders had no net weight change. Fifty-nine responders reported a net weight gain, ranging from 2 to $70 \mathrm{lbs}$ with a mean net weight gain of $16.07 \mathrm{lbs}$ $\pm 13.87 \mathrm{lbs}$. The duration of time since discontinuing PTM use had a significant correlation with weight maintenance: $r=0.340, p$ value $=.000$. There was no significant correlation between duration of time using PTM and weight maintenance. No correlations were identified between education, income, or ethnicity and weight maintenance. A correlation was identified between weight maintenance and current exercise level: $r=0.218, p$ value $=.000$. Conclusions: Although more research is needed, with the results of this study, the investigators suggest that post-PTM use, weight regain is not significantly higher than weight regain through other weight loss programs. In order to achieve long-term weight loss maintenance, it may behoove patients to receive professional guidance about behavior modifications to maintain weight lost, particularly related to exercise and dietary changes. The investigators of this study suggest greater emphasis on behavior modification in patients taking PTM is indicated.
\end{abstract}

\section{Author Bio(s)}

Cassandra Steadman Towns, RDN, LDN, CD is the Whole Health Program Manager at the Tomah Veteran Affairs Medical Center. She earned her Bachelor's in Dietetics from the University of Wisconsin Stevens Point and her Master's in Nutrition and Dietetics from Central Michigan University.

John V. Logomarsino,PhD, RD, LD/N is Professor Emeritus in Nutrition and Dietetics at Central Michigan University. He has 45 years of experience in teaching and research on subjects relating to nutrition science, nutrition education, and health.

Roschelle Heuberger, PhD, RD, is a Professor of Nutrition at Central Michigan University. She completed her doctoral studies and post-doctoral fellowship at the University of Wisconsin- Madison in Nutritional Sciences and Nutritional Epidemiology. She currently sits on the Board for the Medical Nutrition Council of the American Society of Nutrition.

Arturo Olivera Jr, MD, is the owner of Arturo Olivera Jr LTD, the Medical Director of Olivera Weight Management S.C., the Chief Section Gastroenterology at Advocate Illinois Masonic Medical Center, and the owner of Ghazanfari \& Olivera Gastroenterology LTD. He is a Clinical Assistant Professor at Loyola University Medical Center. 


\title{
1IVAHSP \\ The Internet Journal of Allied Health Sciences and Practice \\ Dedicated to allied health professional practice and education \\ Vol. 18 No. 1 ISSN 1540-580X
}

\section{Weight Changes Post-Phentermine Use}

\author{
Cassandra S. Towns ${ }^{1}$ \\ Roschelle A., Heuberger ${ }^{1}$ \\ Jack Logomarsino ${ }^{1}$ \\ Arturo Olicera Jr. ${ }^{2}$
}

1. Central Michigan University

2. Loyola University

United States

\begin{abstract}
Purpose: Evaluate weight maintenance in subjects who lost weight while taking phentermine (PTM). Methods: This study was conducted by utilizing a validated survey to collect self-reported data about weight changes post-PTM use. The survey was sent to 19,986 email addresses of current and former patients of a PTM-based outpatient clinic, located in Chicago, IL; 546 participants completed the survey. Results were analyzed using a variety of techniques. Results: At the time of the survey, $42 \%$ of responders maintained all the weight lost while on PTM, and $25.2 \%$ had maintained a weight loss of at least $10 \%$ of their body weight. The average weight lost and maintained at the time of the survey was $3.75 \% \pm 13.8 \%$ of body weight or $8.74 \mathrm{lbs} \pm 27.45 \mathrm{lbs}$. Nineteen responders had no net weight change. Fifty-nine responders reported a net weight gain, ranging from 2 to $70 \mathrm{lbs}$ with a mean net weight gain of $16.07 \mathrm{lbs} \pm 13.87 \mathrm{lbs}$. The duration of time since discontinuing PTM use had a significant correlation with weight maintenance: $r=0.340, p$ value $=.000$. There was no significant correlation between duration of time using PTM and weight maintenance. No correlations were identified between education, income, or ethnicity and weight maintenance. A correlation was identified between weight maintenance and current exercise level: $r=0.218, p$ value $=.000$. Conclusions: Although more research is needed, with the results of this study, the investigators suggest that post-PTM use, weight regain is not significantly higher than weight regain through other weight loss programs. In order to achieve long-term weight loss maintenance, it may behoove patients to receive professional guidance about behavior modifications to maintain weight lost, particularly related to exercise and dietary changes. The investigators of this study suggest greater emphasis on behavior modification in patients taking PTM is indicated.
\end{abstract}

Keywords: weight loss, phentermine, maintenance, weight maintenance, post-phentermine, pharmacological weight loss

\section{Conflict of Interest Statement/Funding}

Two of the investigators for this study had affiliations with the weight loss clinic at the time of the study. There were no formal funding sources for this study. 


\section{INTRODUCTION}

Increases in obesity rates have slowed, but obesity remains one of the biggest health concerns in the nation. ${ }^{1}$ Over one third of adults in the United States are obese. ${ }^{2}$ People with obesity are more likely to develop chronic medical conditions, such as diabetes, hypertension, sleep apnea, osteoarthritis, and some cancers; they are also more likely to suffer from cardiac complications. ${ }^{2-4}$ Beyond the medical consequences of obesity, studies show evidence of a decreased health-related quality of life. ${ }^{5}$ With such a high proportion of the US population suffering with this disease, more effective interventions for long-term weight loss are needed.

A multitude of strategies and tools can promote weight loss, but many people struggle to maintain the weight they have lost. Roughly, only 1 in every 6 adults in the United States who has been obese or overweight has achieved long-term weight-loss of greater than $10 \%$ of their body weight for at least 1 year. ${ }^{6}$ As a result, more information regarding weight loss maintenance following different modalities of weight loss is indicated.

Several pharmacological agents are being explored to determine their efficacy in weight loss. Currently, 9 Food and Drug Administration (FDA) approved weight loss medications are on the market. ${ }^{7}$ Phentermine (PTM) is the most widely prescribed weight loss medication. ${ }^{8}$ It was approved for short-term use by the FDA in 1959. PTM is a sympathomimetic amine with pharmacologic properties similar to amphetamines. The mechanism of action is seemingly secondary to central nervous system effects, inducing stimulation of the hypothalamus to release norepinephrine. The increased peripheral norepinephrine decreases appetite and may increase energy expenditure. ${ }^{9}$ Although PTM (in conjunction with diet and exercise), is effective in yielding significantly greater weight loss than diet and exercise alone, minimal published literature is available regarding weight

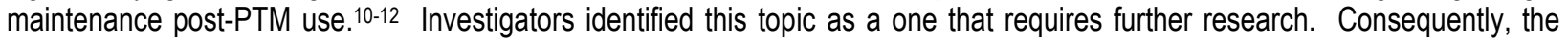
investigators strive to evaluate weight maintenance in subjects who lost weight while taking phentermine.

\section{METHODS}

The survey was deemed internal review board (IRB) exempt by Central Michigan University IRB and approved by the weight loss clinic's IRB. This study was conducted utilizing a validated, emailed survey to collect self-reported data about weight changes postPTM use.

The investigators drafted a 19-question survey that was designed to obtain the following: basic demographic information, anthropometric data, changes in weight during phentermine use, and weight changes post-phentermine use. With the survey, time frames were established during which patients used PTM and then provided basic questions for the following: dietary changes, exercise changes, medical diagnosis, and medication changes. This survey was validated via 3 focus groups; one interdisciplinary focus group and 2 lay (non-health care) focus groups. Prior to the start of the survey, participants were notified that all their information would be de-identified, and that no personal identifying information would be stored. It was made clear that the answers to the survey would be confidential, anonymous, and HIPAA protected. No IP addresses were retained. Participants were notified that the data collected would be analyzed and results published in a research article. Informed consent was implied by the entry into the online survey.

\section{Recruitment (Focus Groups for Survey Validation)}

A formal electronic invitation for participation was sent to the investigators' professional network for the interdisciplinary focus group. An electronic invitation for participation was sent to individuals from different age groups and professional backgrounds for the lay focus group. The invitation included the purpose of the focus group, what was expected of focus group participants, the requirements of participation and additional details.

\section{Data Collection (Survey Validation)}

Each focus group was facilitated utilizing open-ended questions and encouraging discussion. The lay focus groups served as a forum for linguistic validation (a process to ensure survey questions are written using the appropriate language for the target population).

The interdisciplinary focus group consisted of 5 participants: 2 registered dietitians and 3 second-year physician assistant students. Two of the students had work experience as certified nursing assistants and one as a medical assistant. Each participant completed a demographic questionnaire and a consent form. All participants were female; efforts to recruit males were unsuccessful. Two participants had their master's degrees while the other three were enrolled in master's degree programs. Despite efforts to recruit participants from a variety of ethnic backgrounds, this focus group was entirely comprised of Caucasians. The focus group was held in a conference room in a medical office building in Chicago, IL. During the focus group session, participants were invited to 
discuss their understanding of each survey question and to evaluate the appropriateness of the answer options as well as the formatting of the question and answers (i.e., a check box vs a free-text box, etc). This group discussed the survey and the questions within the survey for 2 hours.

The lay focus group consisted of 7 participants: a corrections department employee, an engineer (project lead), an insurance professional, a manufacturing employee, a running coach/personal trainer, a social worker (no health care experience), and a teacher's assistant. Each participant completed a demographic questionnaire and consent. The group included 3 males and 4 females. The age of participants ranged from early 20 s to late 60 s with 2 of the participants being retired. The following ethnicities were represented in this group: Caucasian and Hispanic. The education level of participants ranged from a general equivalency diploma (GED) to a master's degree. The focus group was held at a café in Zion, IL. During the focus group session, participants were invited to discuss their understanding of each survey question and to evaluate the appropriateness of the answer options as well as the formatting of the question and answers (i.e., a check box vs a free-text box, etc.). This group discussed the survey and the questions within the survey for 2.5 hours.

Upon the completion of the lay focus group, the participants were invited to stay at the café and participate in a third focus group, discussing a draft of the survey that included revisions based on the feedback from the interdisciplinary group, survey version 1.2. Five of the participants ( 3 female and 2 male) opted to participate. These 5 participants included the corrections department employee, engineer, manufacturing employee, running coach, and teacher's assistant. During the focus group session, participants were invited to discuss their understanding of each survey question and to evaluate the appropriateness of the answer options as well as the formatting of the question and answers (i.e., a check box vs a free-text box, etc). The group discussed the survey and the questions within the survey for 1 hour.

\section{Data Analysis (Survey Validation)}

Each focus group was recorded using two separate recording devices. The recordings were transcribed (de-identified) and uploaded into NVIVO 11. Each transcription was analyzed individually. NVIVO 11 was used to code all focus group statements and identify common themes. For each question, statements were coded as agree or disagree with the original wording of the question. For all statements coded as disagree, further coding was performed to identify proposed changes. Each statement regarding a proposed change was coded as agree or disagree. Based on this analysis and professional judgement, revisions were made to each survey question to incorporate the most popular recommendations that were in keeping with the survey's intent and design.

NVIVO 11 was used to create visual representations of the focus group findings. As demonstrated by the word clouds, internal consistency was achieved across all three focus groups. Figure 1 is a word cloud representation from the interdisciplinary focus group reviewing survey version 1.1. Figure 2 is a word cloud representation from the lay focus group reviewing survey version 1.1. Figure 3 is a word cloud representation of the second lay focus group reviewing survey version 1.2.

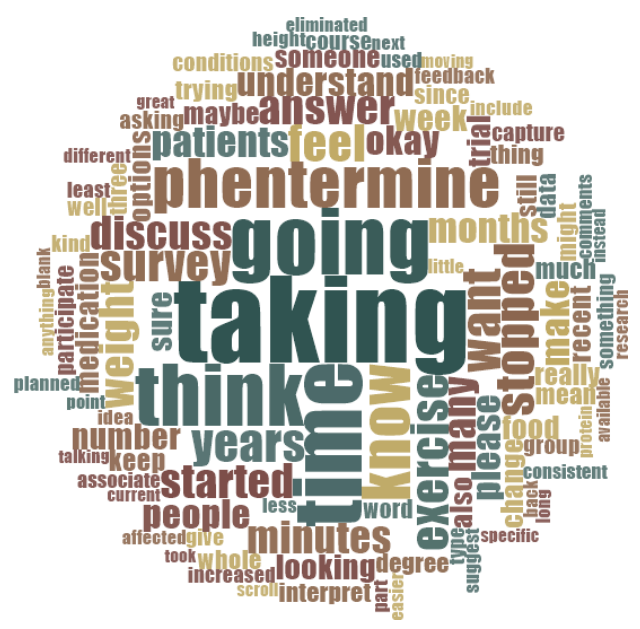

Figure 1. Interdisciplinary Focus Group Survey Version 1.1

(C) The Internet Journal of Allied Health Sciences and Practice, 2020 


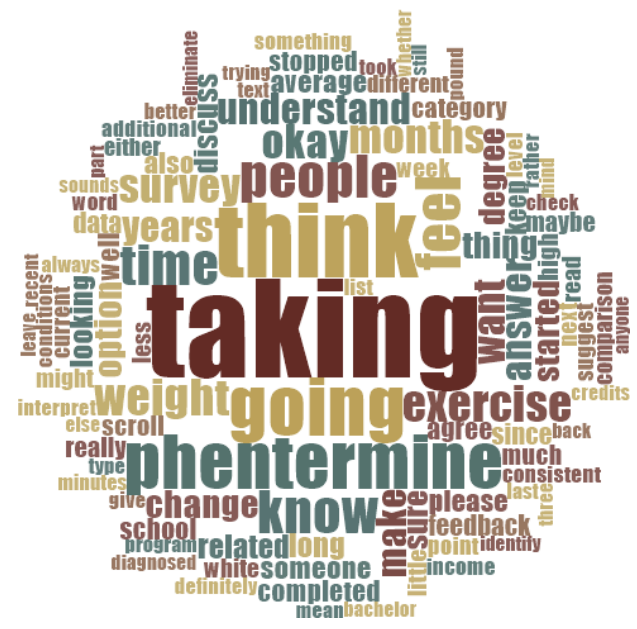

Figure 2. Lay Focus Group Survey Version 1.1

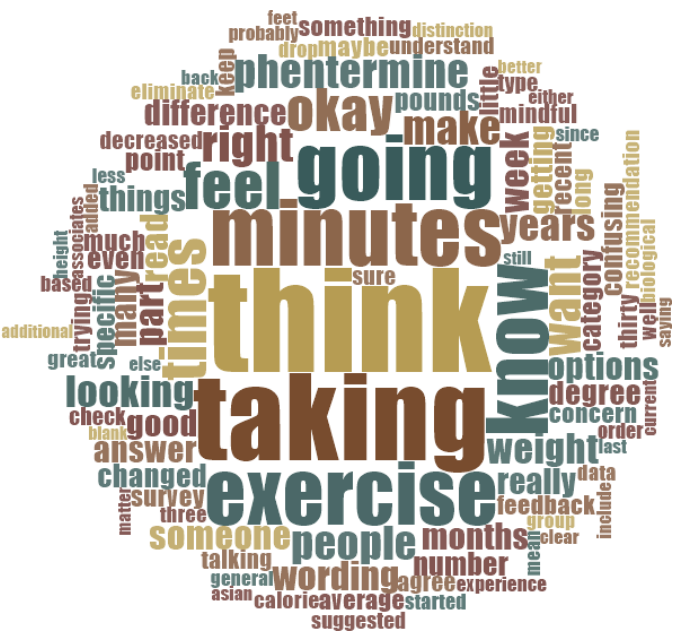

Figure 3. Lay Focus Group Survey Version 1.2

A few strong themes were present in all three focus groups: reinforcement of "in your most recent experience taking phentermine" in each question when appropriate; re-ordering the survey questions to be in a timeline format; bolding time references (ie, before, during, and after); being very clear and concise; and formulating questions to be as simple as possible. Each strong theme was incorporated into the survey.

Survey questions were built into Survey Monkey. Each question was built according to the suggestions from the focus groups (text box vs scroll box, etc). To submit the survey, participants were required to respond to every question. Survey functionality was tested by investigators by emailing the survey and physically taking it.

\section{Data Collection (Survey Distribution)}

The validated survey was sent to 19,986 email addresses of current and former patients of a PTM-based outpatient clinic, located in Chicago, IL. The recruitment emails described the survey and the criteria for participation: having taken PTM for at least one month, at least one month had passed since they discontinued PTM use, and there was a loss of at least 5 lbs while taking PTM. No IP addresses or other personal, identifying information was collected. Reponses were anonymous and confidential. A total of 3 recruitment emails were sent to each of the 19,986 email addresses, resulting in 546 completed surveys. 


\section{Data Analysis (Survey Results)}

Investigators utilized Microsoft Excel to clean the data and SPSS (IBM Corp. Released 2016. IBM SPSS Statistics for Windows, Version 24.0. Armonk, NY: IBM Corp.) to code and analyze the data. Descriptive statistics and frequencies were performed using SPSS to describe the data collected. A variety of analyses were performed, including Spearman and Pearson correlations, chisquared tests, $t$ tests, and regressions.

\section{RESULTS}

\section{Survey Responses}

The survey was sent to 19,986 people via email; 546 people took the survey for a $2.7 \%$ response rate. Of the 546 surveys, 59 surveys were excluded based on reported medical diagnosis or changes in medications that may have affected weight. Of the remaining pool, 201 were deemed non-responders as the survey responses indicated that the responder did not qualify to take the survey. The following exclusion criteria were applied: there was no weight loss of at least $5 \mathrm{lbs}$ while on PTM, had not taken PTM for at least one month, or had not discontinued PTM use at least 1 month prior to taking the survey. Consequently, 286 surveys remained, which were deemed responders. The results of $t$ tests and chi-squared analyses indicated no statistically significant differences between the responders and non-responders; the results reported are from the responder population.

\section{Basic Demographic Information}

The responders consisted of 269 females and 17 males. Their age ranged from 20 to 65 years with an average age of $42.5 \pm 9.19$ years. Of the responders, $36.4 \%$ identified themselves as African-American, $30.8 \%$ identified themselves as Hispanic, $29.4 \%$ identified themselves as Caucasian, and $3.4 \%$ identified themselves as another ethnicity. At the time they started taking PTM, the weight of the responders ranged from 130 to $410 \mathrm{lbs}$ with the average weight being $211.1 \pm 47.5 \mathrm{lbs}$. Their heights ranged from 57 to 75 inches with an average height of $64.87 \pm 3.11$ inches. Average BMl when starting PTM was $35.5 \pm 6.2 \mathrm{~kg} / \mathrm{m} 2$. Additional demographics can be found in Table 1.

Table 1. Additional Demographic Information

\begin{tabular}{lll}
\hline Education Level & Results \\
\hline Highest Education Level & Percent & Frequency \\
Less than a HS diploma & $1.7 \%$ & 5 \\
Obtained their high school diploma but no additional education & $12.6 \%$ & 36 \\
Obtained education beyond high school but less than that of a bachelor's degree & $44 \%$ & 126 \\
Bachelor's degree & $21.7 \%$ & 62 \\
Post-graduate education & $19.9 \%$ & 57 \\
& & \\
Annual Income & Percent & Frequency \\
less than $\$ 20,000$ & $8 \%$ & 23 \\
$\$ 20,000-39,999$ & $16 \%$ & 46 \\
$\$ 40,000-59,9000$ & $25 \%$ & 71 \\
$\$ 60,000-79,999$ & $15 \%$ & 43 \\
$\$ 80,000-99,000$ & $14 \%$ & 40 \\
more than $\$ 100,000$ & $22 \%$ & 63 \\
\hline
\end{tabular}

\section{Reported Phentermine Use}

The duration of time PTM was taken and the duration of time since PTM use was discontinued can be viewed in Table 2. 
Table 2. Phentermine Use

\begin{tabular}{lll}
\hline Duration & Results & \\
\hline Duration on phentermine & Percent & Frequency \\
$>1$ month but $<3$ months & $50.3 \%$ & 144 \\
$>3$ months but $<6$ months & $25.5 \%$ & 73 \\
$>6$ months but $<9$ months & $9.8 \%$ & 28 \\
$>9$ months but $<12$ months & $7.0 \%$ & 20 \\
$>12$ months & $7.3 \%$ & 21 \\
& & \\
Duration of time from discontinuing phentermine to taking the survey & Percent & Frequency \\
$>1$ month but $<3$ months & $28.0 \%$ & 80 \\
$>3$ months but $<6$ months & $12.6 \%$ & 36 \\
$>6$ months but $<9$ months & $15.4 \%$ & 44 \\
$>9$ months but $<12$ months & $10.5 \%$ & 30 \\
$>12$ months but $<18$ months & $10.1 \%$ & 29 \\
$>18$ months but $<24$ months & $6.3 \%$ & 18 \\
$2-3$ years & $8.0 \%$ & 23 \\
$4-5$ years & $4.9 \%$ & 14 \\
$>5$ years & $4.2 \%$ & 12 \\
\hline
\end{tabular}

\section{Exercise}

At the time of the survey, most responders $(60.5 \%, 173$ responders) had not increased their exercise frequency from their baseline. Twenty one percent (60) responders reported exercising less after using PTM than they did before taking PTM. There were $18.5 \%$ (53) responders who reported higher post-PTM use exercise frequency. Reported exercise frequencies before, during, and after PTM use can be seen in Figure 4.

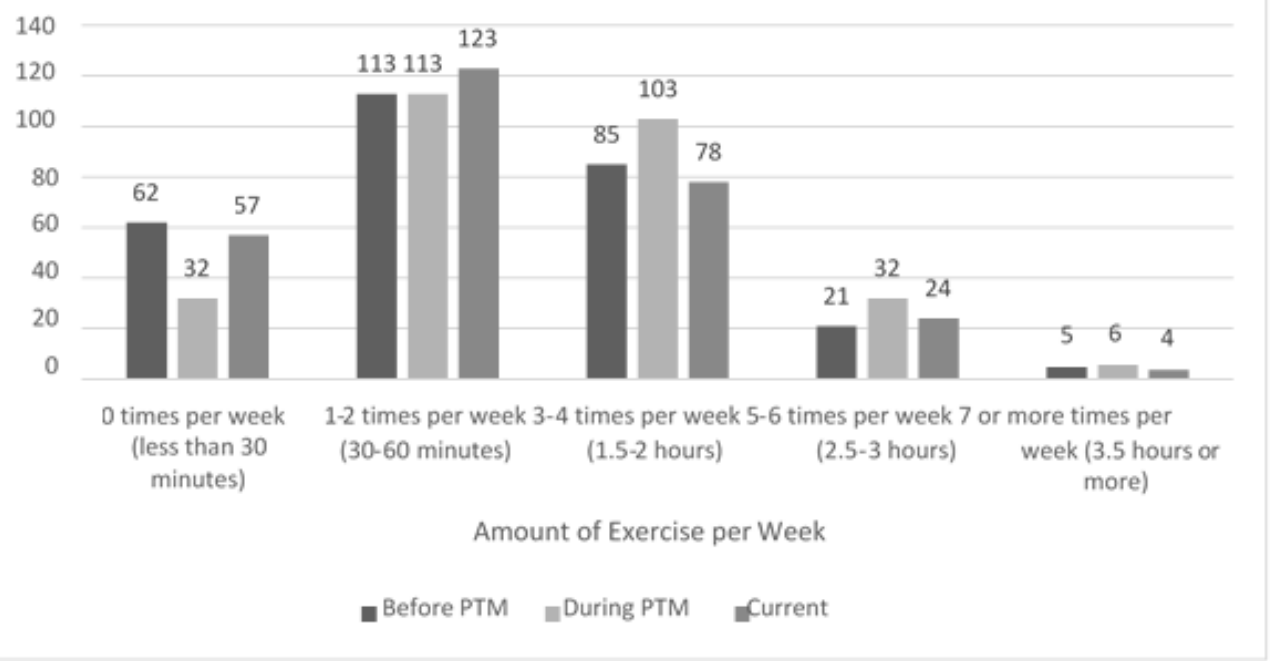

Figure 4. Exercise Before Phentermine Use, During Phentermine Use, and Currently

\section{Dietary Changes}

Several participants reported making changes in their dietary habits during PTM use. Table 3 illustrates those changes in addition to those dietary behaviors that were maintained after PTM use. 
Table 3. Dietary Changes Reported During PTM Use and Changes Maintained Post-PTM Use

\begin{tabular}{lllll}
\hline Change Reported & $\begin{array}{l}\text { During PTM Use } \\
\text { \# of Responders }\end{array}$ & $\begin{array}{l}\text { During PTM Use } \\
\text { \% Responders }\end{array}$ & $\begin{array}{l}\text { Maintained after } \\
\text { d/c PTM } \\
\text { \# of Responders }\end{array}$ & $\begin{array}{l}\text { Maintained after d/c } \\
\text { PTM }\end{array}$ \\
\hline Changed nothing & 30 & 10.5 & 114 & 39.9 \\
Switched to vegetarian diet & 5 & 1.7 & 2 & 0.7 \\
Calorie counted & 83 & 29 & 42 & 14.7 \\
Decreased alcohol intake & 74 & 25.9 & 46 & 16.1 \\
Decreased carbohydrate intake & 136 & 47.6 & 77 & 26.9 \\
Decreased salty/dessert/"junk" foods & 135 & 47.2 & 61 & 21.3 \\
Decreased frequency of eating & 108 & 37.8 & 38 & 13.3 \\
Decreased fast food intake & 136 & 47.6 & 63 & 22 \\
Decreased fat intake & 82 & 28.7 & 43 & 15 \\
Decreased portion sizes & 126 & 44.1 & 56 & 19.6 \\
Decreased sugar-sweetened beverage intake & 94 & 32.9 & 55 & 19.2 \\
Eliminated dairy & 13 & 4.5 & 5 & 1.7 \\
Eliminated gluten & 5 & 1.7 & 4 & 1.4 \\
Eliminated pork and beef & 16 & 5.6 & 14 & 4.9 \\
Increased cooking & 73 & 25.5 & 38 & 13.3 \\
Increased frequency of eating & 10 & 3.5 & 18 & 6.3 \\
Increased fiber intake & 31 & 10.8 & 18 & 6.3 \\
Increased fruit intake & 51 & 17.8 & 26 & 9.1 \\
Increased protein intake & 48 & 16.8 & 23 & 8.0 \\
Increased nonstarchy vegetable intake & 63 & 22 & 30 & 10.5 \\
Increased water intake & 115 & 40.2 & 59 & 20.6 \\
Switched to whole grains & 18 & 6.3 & 8 & 2.8 \\
Other changes reported & 6 & 2.1 & 12 & 4.2 \\
After d/c PTM, included additional changes & - & - & 6 & 2.1 \\
\hline
\end{tabular}

\section{Weight Loss Findings}

Weight lost by subjects ranged from 5 to $100 \mathrm{lbs}$. The average weight loss while taking PTM was $19.58 \mathrm{lbs} \pm 13.92 \mathrm{lbs}$. Mean weight loss of all responders was $8.23 \% \pm 7.79 \%$ of body weight. There were $37.8 \%$ (108) of subjects who lost at least $10 \%$ of their body weight while taking PTM. Spearman and Pearson correlations were completed, evaluating the correlation between weight lost and all other information collected. A positive correlation was identified between weight loss and weight when starting PTM use $(r=0.312, p$ value $=.000)$, and between starting BMl and weight loss $(r=0.353, p$ value $=.000)$. An inverse correlation between weight lost and age was identified: $r=-0.140, p$ value $=.01$, indicating that younger subjects were more likely to have lost a greater amount of weight. Duration of time on PTM had a positive correlation with weight loss: $r=0.239, p$ value $=.000$, indicating that responders who took PTM for a greater duration of time were more likely to lose a greater amount of weight. Negative correlations were identified between 5 reported dietary changes while taking PTM and the amount of weight lost: calorie counting $(r=-0.129, p$ value $=.029)$, decreased fast food intake $(r=-0.134, p$ value $=.024)$, decreased frequency of eating $(r=-0.186, p$ value $=.002)$, elimination of dairy $(r=-0.123, p$ value $=.038)$, and increased cooking $(r=-0.121, p$ value $=.040)$. No positive correlations were identified between weight loss and specific dietary changes or the number of dietary changes implemented. Lastly, the amount of exercise during PTM use had a positive correlation with weight loss: $r=0.124, p$ value $=.036$.

\section{Maintenance Findings}

At the time of the survey, $42 \%$ of responders maintained all of the weight lost while on PTM, and $25.2 \%$ had maintained a weight loss of at least $10 \%$ of their body weight. The average weight lost and maintained at the time of the survey was $3.75 \% \pm 13.8 \%$ of body weight or $8.74 \mathrm{lbs} \pm 27.45 \mathrm{lbs}$. Nineteen responders had no net weight change. Fifty-nine responders reported a net weight gain, ranging from 2 to $70 \mathrm{lbs}$ with a mean net weight gain of $16.07 \mathrm{lbs} \pm 13.87 \mathrm{lbs}$.

The duration of time since discontinuing PTM use had a significant correlation with weight maintenance: $r=0.340, p$ value $=.000$. Over half of those who reported a net weight gain (50.8\%, 30 responders) discontinued PTM use between 1 and 5 years prior to taking the survey with just $11.9 \%$ (7 responders) having discontinued PTM between 1 and 3 months prior to taking the survey. There was no significant correlation between duration of time on PTM and weight maintenance. 
Of note, no correlations were identified between education, income, or ethnicity and weight maintenance. There was no significant correlation between maintaining $10 \%$ weight loss and BMI when starting PTM. A highly significant correlation was identified between weight maintenance and current exercise level: $r=0.218, p$ value $=.000$ ). There were no significant correlations between the level of exercise while on PTM and weight maintenance. Positive correlations were identified between three dietary changes made while taking PTM and weight maintenance: the elimination of gluten $(r=0.125, p$ value $=.035)$, decreased frequency of eating $(r=0.140, p$ value $=.018)$ and calorie counting $(r=0.118, p$ value $=.046)$. Additionally, the number of dietary changes maintained after discontinuing PTM had a positive correlation: $r=0.127, p$ value $=.032$. Negative correlations between weight maintenance and dietary changes maintained can be viewed in Figure 5.

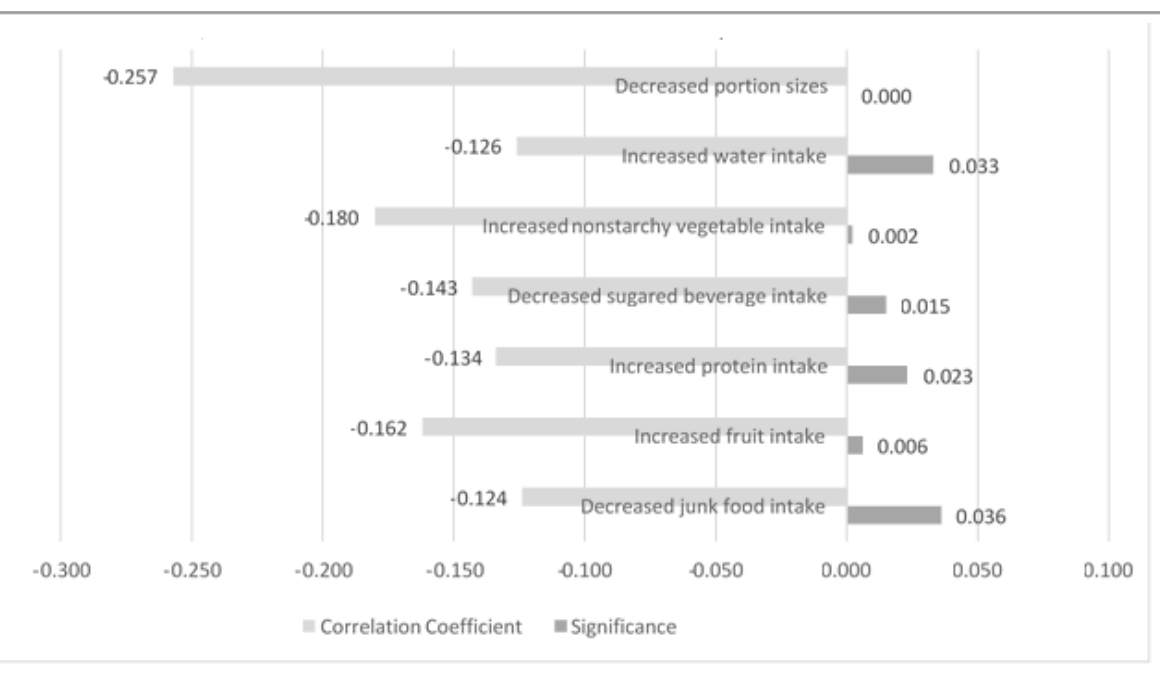

Figure 5. Negative Correlations: Weight Maintenance and Dietary Changes Maintained After Discontinuing

Further analysis of the $25.2 \%$ of responders who had maintained a weight loss of at least $10 \%$ of their body weight was completed. There was no significant correlation between maintaining $10 \%$ weight loss and BMI when starting PTM. Three dietary changes during PTM use had negative correlations with maintaining 10\% weight loss: decreased fat intake $(r=-0.142, p$ value $=.016)$, increased non-starchy vegetable intake $(r=-0.144, p$ value $=.015)$, and increased protein intake $(r=-0.149, p$ value $=.012)$. There was no correlation between maintaining $10 \%$ of body weight loss and income or education level. Duration of time on PTM had a positive correlation with maintaining $10 \%$ of body weight loss $(r=0.154, p$ value $=.005)$ while duration of time since discontinuing PTM had a negative correlation $(r=-0.102, p$ value $=.049)$. Exercise while taking PTM and current level of exercise had a positive correlation with maintaining $10 \%$ weight loss $(r=0.125, p$ value $=.023, r=0.111, p$ value $=.043$, respectively). Positive correlations between dietary changes maintained after discontinuing PTM use and maintaining $10 \%$ of body weight loss can be viewed in Figure 6. 


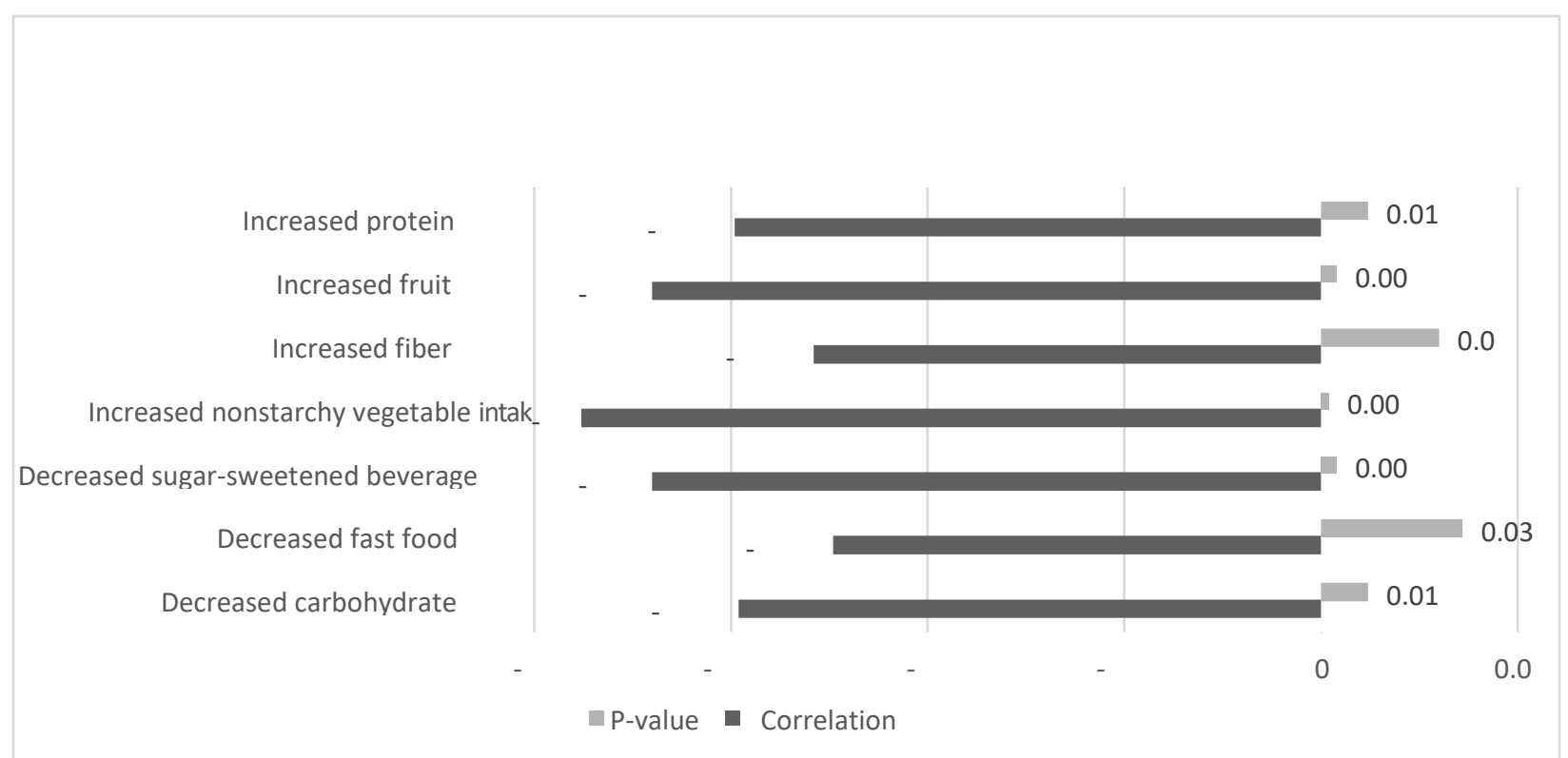

Figure 6. Dietary Changes Maintained After Discontinuing Phentermine Correlated with Maintaining 10\% Body Weight Loss

\section{Contributions of Age, Exercise, Diet, and Duration of Phentermine Use to Weight Outcomes}

Multiple linear regressions and logistic regressions were run, using SPSS v.24, AMOK, NY. Models were run in a stepwise manner. Constants were determined by the software for all models. Several models for logistic regressions showed that weight lost was significantly related to exercise, duration of PTM use, and number of dietary changes maintained. Additionally, $10 \%$ of weight loss against the number of dietary changes maintained after discontinuation of PTM, and the age of the respondent explained $8.7 \%$ of the variability. When current exercise was added to this model, the variability explained increased to $15 \%$. In a separate logistic regression adding duration of time of discharge, PTM against maintaining a 10\% weight loss showed significance for age, decreased sugar sweetened beverage intake, and current exercise levels. Percent of body weight lost and maintained was significant for current exercise level, age, and number of dietary changes maintained after discontinuing PTM and the duration of time since discontinuing PTM, accounting for $12 \%$ variability. Age was consistently significant in each of the models tested. Older age reflected poorer outcomes in terms of maintenance of weight lost during the intervention. Success was tied to behavioral changes made and longitudinal variables as was to be expected.

\section{DISCUSSION}

The investigators examined self-reported weight changes after discontinuing PTM in subjects who had taken PTM for at least one month, had lost at least $5 \mathrm{lbs}$ while taking PTM and who had discontinued use at least 1 month prior to taking the survey. Data were collected via a validated survey distributed to an email list of patients from a PTM-based weight-loss clinic.

Analysis of survey responses indicated $37.8 \%$ of responders had lost $10 \%$ or less of their body weight while taking PTM with $25.2 \%$ of responders having maintained weight loss of $10 \%$ or less of their body weight at the time of the survey. Weight loss and maintenance of $10 \%$ or less of body weight can be beneficial to blood glucose, cholesterol, and blood pressure and decreases the risk of chronic diseases associated with obesity and, therefore, is clinically significant. ${ }^{13}$ In the medical community, it is commonly assumed patients will quickly regain the weight they lost while taking a prescription weight loss medication and possibly gain additional weight. The results of this survey do not support that assumption. On average, subjects maintained a total weight loss of $3.75 \% \pm 13.8 \%$ of their body weight or $8.74 \pm 27.45 \mathrm{lbs}$. Subjects were fairly successful in losing weight and maintaining at least some of the weight loss. There were $20.6 \%$ of responders (59) who experienced an overall net weight gain, ranging from 2 to 70 Ibs with a mean net weight gain of $16.07 \pm 13.87 \mathrm{lbs}$. The duration of time since discontinuing PTM use was significantly correlated to weight maintenance $(r=0.340, p$ value $=.000)$. Over half of the responders who reported a net weight gain discontinued PTM use between 1 and 5 years prior to taking the survey. Only $2.4 \%$ (7) of responders who experienced a net weight gain had discontinued PTM between 1 and 3 months prior to taking the survey. It appears that few responders (10.1\%) who had discontinued PTM within the last year experienced a net weight gain. This weight gain is more likely associated with negative behavior 
modifications and lack of physical activity than with the experience of having taken PTM.

The positive correlation between weight when starting PTM and weight loss indicated that those with higher starting weights were likely to lose a greater amount of weight. Additionally, BMI was positively correlated with weight loss, $r=0.353, p$ value $=.000$, further confirming this indication.

Duration of time taking PTM did not have a significant correlation with weight maintenance, indicating that subjects who took PTM for a longer period of time were no more or less likely to experience weight regain. Duration of time on PTM did have a positive correlation with maintaining $10 \%$ of body weight loss, indicating that for those who lost $10 \%$ of their body weight, the longer they were on PTM, the more likely they were to keep the weight off. The duration of time since discontinuing PTM had a negative correlation with maintaining $10 \%$ weight loss, indicating that the longer one has been off PTM, the more likely one is to have regained weight. This weight gain is to be expected with any weight loss program, and length of time in the maintenance phase was positively correlated with weight regain throughout this study.

Despite generally known demographic correlations with excess body weight, no significant correlations were identified between education, income, or ethnicity and weight maintenance in this population. Weight maintenance and the maintenance of $10 \%$ body weight loss following PTM use appear to be unaffected by these demographic factors.

Before, during, and after PTM use, responders of this survey participated in exercise far below the quantity advised by national recommendations (see Figure 4). ${ }^{14}$ Before PTM use, $90.9 \%$ of responders (260) were not meeting the recommendation of 150 minutes per week (for weight maintenance). During PTM use, $86.7 \%$ (248) of responders were not meeting physical activity guidelines for weight maintenance with no more than $2.1 \%$ (6) responders meeting the recommendation of 300 minutes of activity per week for weight loss. After PTM use, $90.2 \%$ of responders (258) were not meeting physical activity recommendations for weight maintenance. When evaluating weight changes post-PTM use, it is important to note that this population failed to meet physical activity recommendations for weight maintenance and weight loss.

At the time of the survey, the majority of responders ( $60.5 \%, 173$ responders) had not increased their exercise frequency from their baseline (see Figure 4). Twenty one percent (60) of responders reported exercising less after using PTM than they did before taking PTM. There were $18.5 \%$ (53) responders who reported higher post-PTM use exercise frequency. It is also important to take this finding into consideration when evaluating weight maintenance post-PTM use. The frequency of exercise during PTM use was positively correlated with weight loss, indicating that increased exercise frequency likely contributed to greater weight loss. Additionally, current exercise frequency was positively correlated weight maintenance, indicating that those who exercised more were more likely to maintain their weight loss. Exercise levels during PTM use and current exercise level were both positively correlated with weight maintenance of $10 \%$ of body weight. Each of these results re-enforces the value of physical activity for weight loss and maintenance of weight loss. There was no significant correlation between maintaining $10 \%$ weight loss and BMI when starting PTM.

The most popular dietary changes implemented during PTM use included (listed in order of frequency) decreased carbohydrate intake, decreased fast food intake, decreased "junk" food intake, decreased portion sizes, increased water intake, and decreased frequency of eating. The responders who received care at the clinic in Chicago received brief, basic diet and exercise recommendations. These changes are generally viewed as simple changes to implement with minimal professional dietary guidance. None of the specific dietary changes implemented had positive correlations with weight loss.

No positive correlations were identified between weight loss and any specific dietary changes or the number of dietary changes implemented. However, 5 negative correlations were identified: calorie counting, decreased fast food intake, decreased frequency of eating, elimination of dairy, and increased cooking. Decreased frequency of eating and calorie counting were both positively associated with weight maintenance, but negatively associated with weight loss. With PTM use, some patients decreased the frequency of eating to a frequency that was detrimental to weight loss (1-2 times per day) and may have resulted in a mildly decreased metabolic rate, contributing to the negative correlation. Additionally, decreased frequency of eating does not necessarily imply a decreased overall caloric intake. Several studies have shown a positive correlation between dairy intake and weight loss. A negative correlation with the elimination of dairy is consistent with those findings. When eliminating dairy, responders may also have replaced it with dairy alternatives and not necessarily have decreased their overall caloric intake.

Although calorie counting implies a decreased caloric intake, patients new to calorie counting may not actually decrease their caloric intake when calorie counting. Subjects who calorie counted may have counted but may not have decreased their caloric 
intake enough to solicit weight loss. Decreased fast food intake and cooking more at home having a negative correlation with weight loss is atypical, but again, neither change necessarily automatically results in a decreased overall caloric intake. Lastly, for each of these changes, the responders may have indicated any changes they made for any amount of time rather than changes they made and maintained the entire time they were taking PTM. For example, a subject may have calorie counted for 2 weeks, but not for the full 6 months they were taking PTM.

Three positive correlations were identified between dietary changes made while taking PTM and weight maintenance: elimination of gluten, decreased frequency of eating, and calorie counting. Also, the number of dietary changes maintained after discontinuing PTM had a positive correlation. When eliminating gluten, commonly less processed foods are consumed. The responders who eliminated gluten from their diets may have switched to a diet containing less calorie-dense processed foods and more whole foods that would explain this correlation. The positive correlation with calorie counting is consistent with expectations if calorie counting is being done correctly. Decreased frequency of eating during PTM use may have resulted in changes in snacking habits while taking PTM, which carried over into the maintenance phase. Three positive correlations were also identified between dietary changes made while taking PTM and maintenance of at least $10 \%$ of body weight: decreased fat intake, increased non-starchy vegetable intake, and increased protein intake. When implemented appropriately, these changes are known to positively affect weight loss and weight maintenance. Surprisingly, there were no dietary changes made during PTM use that were both positively, significantly correlated with weight maintenance and the maintenance of $10 \%$ body weight.

Negative correlations between dietary changes maintained after discontinuing PTM use and weight maintenance included decreased portion sizes, increased water intake, increased non-starchy vegetable intake, decreased sugar sweetened beverage intake, increased protein intake, increased fruit intake, and decreased junk food intake (see Figure 5). These maintained dietary changes were each negatively correlated with maintaining at least $10 \%$ weight loss as well (see Figure 6 ). Each of these individual changes were very specific, and although each of them may contribute to an overall healthier, lower calorie diet, they do not necessarily imply significant enough change to result in a positive correlation with weight loss or weight maintenance. For example, if fruit, vegetables, and protein are added, but nothing else is removed from a diet, caloric intake will actually increase, which would explain the negative correlation. Additionally, some of the responders who indicated that they maintained these changes post PTM use may have implemented these changes between the time they stopped taking PTM and the time they took the survey. They could have regained weight, then added these changes.

A number of different logistic regression models and the models that came up significant contributed between $8 \%$ and $15 \%$ variability. Exercise was consistently significant with weight maintenance of $10 \%$ body weight and overall maintenance of weight lost. Age was also consistently significant with older adults less able to maintain their weight loss percentage over time.

Despite the multiple regressions, only low percentages explaining variability were identified and the remaining variability could not be accounted for. This variability could be due to measurement error or a number of other factors, such as the therapeutic relationship with the provider, variability in behavior modification recommendations, etc. Seventy-seven percent of participants in the National Weight Control Registry who have maintained a weight loss of at least 30 lbs for at least 1 year reported a triggering event. ${ }^{15}$ If a responder had experienced a triggering event, it could have been a significant confounder in this study.

\section{Strengths and Weaknesses of this Study Strengths}

- The responders of this survey included a variety of demographic backgrounds. Roughly one third identified themselves as African-American, one third Hispanic, and one third Caucasian, making the results of this survey representative of a wider ethnic background than typically found in weight loss surveys. Age ranged from 20 to 65 years. Income level ranged from below the poverty line to six figures. Education ranged from less than a high school diploma to doctoral degrees. As a result, the results of this survey are representative of a large demographic.

- The survey utilized in this study was validated through three focus groups and piloted. It required responses to all questions, so every survey submitted was complete. The results of $t$ tests and chi-squared analyses indicated no statistically significant differences between the responders and non-responders. Survey questions provided coverage of many behavioral interventions and confounders that might influence weight loss.

- After identifying the responders of this survey, the sample size was sufficient.

- The investigators tried to account for biases, although some may still have been present.

- All responders came from the same PTM-based clinic in Chicago and were on the same program. 


\section{Weaknesses}

- This study had a cross-sectional survey; no causality is implied.

- Due to the poor response rate, participation bias is possible.

- Several variables were contrived.

- $\quad$ Although the survey was validated via three focus groups and analysis utilizing INVIVO 11, investigators identified one response option to a question that likely confounded the results. When asked "what dietary changes have you maintained," one of the response options was "nothing changed." Rather than identifying specifically which changes were maintained, this option was selected. Ideally, nothing changed would have remained a response for the question "what dietary changes did you make while taking PTM," but not when asking "what dietary changes have you maintained."

- The demographic of patients who frequented the clinic population that this survey was sent to were predominantly female, and so were the responders of this survey. With just 17 males having taken the survey, the results were likely not representative of the male population.

- The responders to the survey all frequented a phentermine-based weight loss clinic in Chicago, IL, so results may not be representative of the general population, but rather to that within the greater Chicago area.

- Data were collected via self-reported survey.

- Measurement errors likely occurred. Due to the survey being voluntary, keeping the number of questions limited was a priority, but likely also resulted in valuable information not being included.

- Some lack of statistical significance be due to categorical responses.

- Recall bias was likely a factor, especially related to weight, dietary changes, and exercise. Recall bias likely accounted for some of the correlations between dietary changes and weight maintenance.

\section{CONCLUSIONS}

With the results of this study, the investigators suggest greater emphasis for behavior modification in patients when taking PTM is indicated. The vast majority of responders in this study failed to meet physical activity guidelines before, during, and after PTM use. In the National Weight Control Registry, $94 \%$ of people who had maintained a weight loss of at least $30 \mathrm{lbs}$ for at least one year increased their physical activity levels while just $18.5 \%$ of responders had increased their exercise levels from pre-PTM use to post-PTM use. ${ }^{16}$

Although more research is needed, the investigators suggest that post-PTM use weight regain is not significantly higher than weight regain through other weight loss programs. At the time of the survey, $25 \%$ of responders had maintained a weight loss of at least $10 \%$ of their body weight. The majority of those responders had maintained that weight loss for between 1 and 3 months. In a study about long-term weight loss maintenance using 1999-2006 National Health and Nutrition Examination Survey data for 14,306 participants, it was found that one sixth of the adults achieved long-term weight loss maintenance of at least 1 year with $17.3 \%$ having maintained a weight loss of $10 \% .{ }^{6}$ As with any evidence-based weight loss modality, duration of time in the maintenance phase was strongly correlated to gradual weight regain. Additional research is indicated, particularly in post-PTM use weight regain in subjects who discontinued PTM at least one year prior to the study.

Studies have shown greater success in long-term weight loss when subjects receive medical nutrition therapy in conjunction with FDA approved weight loss medications than with medication alone. ${ }^{17}$ The investigators suggest that it would behoove patients to receive professional guidance for behavior modifications, specifically those diet and exercise related. This conclusion is consistent with current treatment recommendations. , $^{9,18-20}$

\section{References}

1. Behavioral Risk Factor Surveillance System, 2016 Survey Data and Documentation. In U.S. Centers for Disease Control and Prevention. https://www.cdc.gov/brfss/data_documentation/index.htm. Published August 29, 2017. Accessed February 18, 2018.

2. National Center for Health Statistics. Centers for Disease Control and Prevention. https://www.cdc.gov/nchs/data/factsheets/factsheet_nhanes.htm. Published December 14, 2017. Accessed February $17,2018$.

3. Expert panel report: Guidelines (2013) for the management of overweight and obesity in adults. Managing Overweight and Obesity in Adults: Systematic Evidence Review from the Obesity Expert Panel. November 2013. doi:10.1002/oby.20660.

4. Bhaskaran K, Douglas I, Forbes H, Dos-Santos-Silva I, Leon DA, Smeeth L. Body-mass index and risk of 22 specific 
cancers: a population-based cohort study of 5·24 million UK adults. The Lancet. 2014;384(9945):755-765. doi:10.1016/s0140-6736(14)60892-8.

5. Lopez-Garcia E, Guallar-Castillón P, Garcia-Esquinas E, Rodríguez-Artalejo F. Metabolically healthy obesity and health-related quality of life: A prospective cohort study. Clinical Nutrition. 2017;36(3):853-860. doi:10.1016/j.clnu.2016.04.028.

6. Kraschnewski JL, Boan J, Esposito J, et al. Long-term weight loss maintenance in the United States. International Journal of Obesity. 2010;34(11):1644-1654. doi:10.1038/ijo.2010.94. [PMID 20479763]

7. Prescription Medications to Treat Overweight and Obesity. National Institute of Diabetes and Digestive and Kidney Diseases. https://www.niddk.nih.gov/health-information/weight-management/prescription-medications-treatoverweight- obesity. Published July 1, 2016. Accessed February 18, 2018.

8. Apovian CM, Aronne LJ, Bessesen DH, et al. Pharmacological Management of Obesity: An Endocrine Society Clinical Practice Guideline. The Journal of Clinical Endocrinology \& Metabolism. 2015;100(2):342-362. doi:10.1210/jc.2014- 3415.

9. Phentermine. In: Post T, ed. UpToDate. Waltham, Mass.: UpToDate; 2018. www.uptodate.com. Accessed February 26, 2018.

10. Ryan DH, Bray GA. Pharmacologic Treatment Options for Obesity: What Is Old Is New Again. Current Hypertension Reports. 2013;15(3):182-189. doi:10.1007/s11906-013-0343-6. [PMID 23625271]

11. VI-0521 (QNEXA®) Advisory Committee Briefing Document. NDA 022580. Endocrinologic and Metabolic Drugs Advisory Committee Meeting. 2012. https://www.nutricity.it/wp-content/uploads/2013/02/Fentermina-Topiramato.pdf

12. Kang JG, Park C-Y, Kang JH, Park Y-W, Park SW. Randomized controlled trial to investigate the effects of a newly developed formulation of phentermine diffuse-controlled release for obesity. Diabetes, Obesity and Metabolism. 2010;12(10):876-882. doi:10.1111/j.1463-1326.2010.01242.x.

13. Blackburn G. Effect of Degree of Weight Loss on Health Benefits. Obesity Research. 1995;3(S2). doi:10.1002/j.15508528.1995.tb00466.x.

14. Appendix 1. Physical Activity Guidelines for Americans. https://health.gov/dietaryguidelines/2015/guidelines/appendix1/. Accessed February 1, 2019.

15. Klem ML, Wing RR, Mcguire MT, Seagle HM, Hill JO. A descriptive study of individuals successful at long-term maintenance of substantial weight loss. The American Journal of Clinical Nutrition. 1997;66(2):239-246. doi:10.1093/ajcn/66.2.239.

16. National Weight Control Registry. http://www.nwcr.ws/Research/default.htm. Accessed February 13, 2019.

17. Mcmahon $\mathrm{K}$, Wengreen $\mathrm{H}$, Schvaneveldt $\mathrm{N}$. Weight and Inch Changes in Subjects Enrolled in a Medical Weight Loss Management Program With and Without Medical Nutrition Therapy. Journal of the American Dietetic Association. 1997;97(9). doi:10.1016/s0002-8223(97)00487-2.

18. Apovian CM, Aronne LJ, Bessesen DH, et al. Pharmacological Management of Obesity: An Endocrine Society Clinical Practice Guideline. The Journal of Clinical Endocrinology \& Metabolism. 2015;100(2):342-362. doi:10.1210/jc.20143415.

19. Mccabe-Sellers BJ. Position of the American Dietetic Association: Integration of Medical Nutrition Therapy and Pharmacotherapy. Journal of the American Dietetic Association. 2010;110(6):950-956. doi:10.1016/j.jada.2010.04.017.

20. Author, Scaling Up Efforts to Bring Weight Down: An Update on Recommendations, Techniques, and Pharmacotherapies for Adult Weight Management. Clinician Reviews. 2015;25(12). 